Association for Information Systems AIS Electronic Library (AISeL)

Wirtschaftsinformatik Proceedings 1999

Wirtschaftsinformatik

February 1999

\title{
Redesign von WWW-basierten Masseninformationssystemen
}

Marion Kaukal

Universität WU Wien, Marion.Kaukal@wu-wien.ac.at

Bernd Simon

UniversitätWU Wien, bernd.simon@wu-wien.ac.at

Follow this and additional works at: http://aisel.aisnet.org/wi1999

\section{Recommended Citation}

Kaukal, Marion and Simon, Bernd, "Redesign von WWW-basierten Masseninformationssystemen" (1999). Wirtschaftsinformatik Proceedings 1999. 4.

http://aisel.aisnet.org/wi1999/4

This material is brought to you by the Wirtschaftsinformatik at AIS Electronic Library (AISeL). It has been accepted for inclusion in Wirtschaftsinformatik Proceedings 1999 by an authorized administrator of AIS Electronic Library (AISeL). For more information, please contact elibrary@aisnet.org. 


\section{Redesign von WWW-basierten Masseninformationssystemen}

\section{Marion Kaukal}

Universität WU Wien (Marion.Kaukal@wu-wien.ac.at)

Bernd Simon

Universität WU Wien (Bernd.Simon@wu-wien.ac.at)

\section{Inhalt}

\section{Einleitung}

2 Maximierung des Anwender-Nettonutzens

3 Das Vorgehensmodell zum Redesign von WMIS

3.1 Maximierung des Anwender-Bruttonutzens

3.1.1 Analyse der Konkurrenz-WMIS

3.1.2 Nachfrageanalyse im eigenen WMIS

3.2 Minimierung des Anwender-Aufwands

3.2.1 Evaluation des Informationsdesigns (Strukturierung des WMIS)

3.2.2 Evaluation des Navigationsdesigns

3.3 Redesign 


\begin{abstract}
Viele WWW-basierte Masseninformationssysteme (WMIS) wurden nicht im Sinne eines systematisch durchgeführten Softwareprojekts mit klar definierten Zielen realisiert. Diese Arbeit richtet sich speziell an Betreiber von WMIS, die bisher sehr unstrukturiert an das Design ihres Informationssystems herangegangen sind.

Das präsentierte Vorgehensmodell für ein Redesign basiert auf einer marketing-orientierten Annäherung an die Problemstellung. Es wird der Begriff "Anwender-Nettonutzen" eingeführt, der aus der Differenz von Anwender-Bruttonutzen und Anwender-Aufwand ermittelt wird. Es wird untersucht, inwieweit der Anwender-Nettonutzen eines WWW-basierten Masseninformationssystems optimiert werden kann. Dabei werden Techniken wie die Konkurrenz-Analyse und die Log-File-Analyse herangezogen.
\end{abstract}

Das Modell schlägt die Definition von Erfolgskriterien vor, die von den Zielen (Soll-Werten) der durch das Informationssystem unterstützten Geschäftsprozesse abgeleitet werden können. Anhand der Erfüllung der Erfolgskriterien kann die Notwendigkeit eines Redesigns festgestellt und unter der Zielsetzung den Anwender-Nettonutzen zu optimieren, durchgeführt werden.

Die grafischen und funktionellen Kriterien des Web-Designs wurden größtenteils außer acht gelassen. Obwohl sie zweifellos ein wichtiges Charakteristikum eines WMIS darstellen, konzentrieren sich die Autoren auf die inhaltlichen Kriterien, da diese von der Literatur meist vernachlässigt werden.

\title{
1 Einleitung
}

Die Umweltbedingungen eines WWW-basierten Masseninformationssystems (WMIS) sind einem ständigen Veränderungsprozeß unterworfen. Während uns die Informationstechnologiebranche regelmäßig mit neuen Standards und neuen Produkten konfrontiert, ändert sich zur selben Zeit auch das Profil der Benutzer. Aus Surfern werden Anwender, die sich nicht mehr damit zufrieden geben, von bunten Web-Sites unterhalten zu werden, sondern möglichst effizient an die gesuchte Information gelangen möchten. Daraus resultieren neue Anforderungen an die WMIS und damit an die Entwicklung und Wartung derselben (vgl. Bichler 1997, S. 97).

Der Beitrag präsentiert ein Vorgehensmodell für das Redesign von WWWbasierten Masseninformationssystemen.

Unter Redesign verstehen die Autoren, angelehnt an die Definition von Web-Site Design nach Powel (Powel et al. 1998, S. 2), die Reorganisation von Information, 
wobei dies auch die graphische Neuaufbereitung des WWW-Angebots und die Anwendungsentwicklung beinhaltet. In dieser Arbeit wird ein Schwerpunkt auf die Reorganisation der angebotenen Information gesetzt. Auf die graphische Neuaufbereitung und die Anwendungsentwicklung soll nicht näher eingegangen werden.

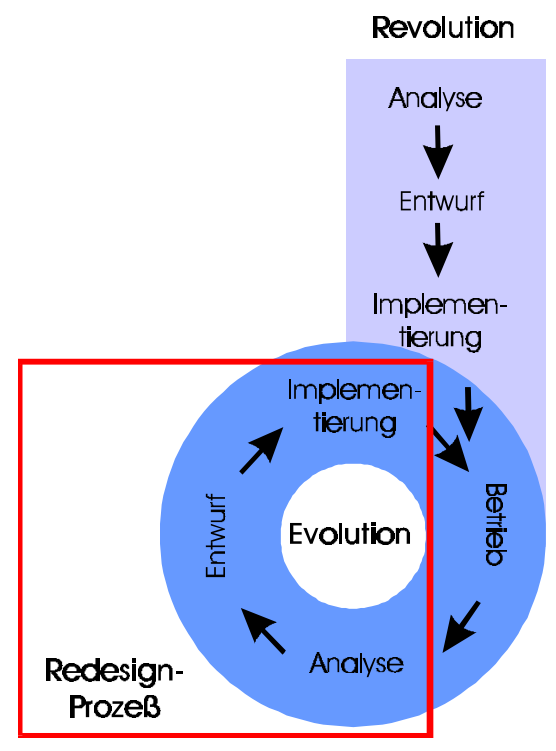

\section{Abbildung 1: Positionierung des Vorgehensmodells in Anlehnung an Österle, Revolution und Evolution (Österle 1995, S. 23)}

Ein Masseninformationssystem dient zur interaktiven Kommunikation eines Betriebes mit potentiell tausenden, hunderttausenden oder millionen privater Kunden beziehungsweise Interessenten, mit denen unter Umständen bisher noch keine oder nur sehr lose gelegentliche Kontakte bestehen (Hansen 1996, S. 412).

Das präsentierte Vorgehensmodell steht unter der Prämisse, den AnwenderNettonutzen eines WWW-basierten Masseninformationssystems zu maximieren. Der Begriff "Anwender-Nettonutzen" wurde vom Marketing-Begriff "Customer Delivered Value" abgeleitet. Der besondere Charakter von Masseninformationssystemen, sie dienen meist dazu, neue Absatzkanäle zu erschließen und die Kommunikation mit den Kunden zu verbessern (vgl. Hansen 1996, S. 412f.), rechtfertigt diese marketing-geleitete Annäherung an die Problemstellung.

Aus der Sichtweise des Business Engineering kann das Vorgehensmodell im Revolution-Evolution-Modell nach Österle in der Entwurfs-, Analyse- und Implementierungsphase des Evolutionsprozesses eingeordnet werden (siehe Abbildung 1). Laut Österle erfordert Business Engineering zuerst ein Projekt (Revolution) und dann die Weiterentwicklung (Evolution) durch die Prozeß- 
führung (Österle 1995, S. 23). Da in der Regel mehrere Geschäftsprozesse von einem WMIS unterstützt werden, besteht die Gefahr, daß die Kunden die Übersicht in der oft gewachsenen Navigationsstruktur verlieren. Damit die Geschäftsprozesse ihre Zielvorgaben erfüllen können, ist die Organisationseinheit "WWWKoordinator" "gefordert, das Masseninformationssystem einem Redesign zu unterziehen.

Der folgende Abschnitt definiert den Begriff "Anwender-Nettonutzen". Anschließend wird auf das Redesign näher eingegangen.

\section{Maximierung des Anwender-Nettonutzens}

Den Begriff "Anwender-Nettonutzen" leiten die Autoren von dem Begriff "Customer Delivered Value" ab, welcher durch die Subtraktion der Total Customer Costs vom Total Customer Value ermittelt wird (vgl. Kotler 1994, S. 552). Analog dazu wird der Anwender-Nettonutzen (ANN) aus dem AnwenderBruttonutzen abzüglich des Anwender-Aufwands berechnet (Für einen Vergleich verschiedener Customer Value-Definitionen siehe Anhang A: Gegenüberstellung von Customer Value-Definitionen).

Um den Anwender-Nettonutzen zu maximieren, muß der Anwender-Aufwand minimiert und der Anwender-Bruttonutzen maximiert werden. Der AnwenderBruttonutzen kann durch eine möglichst optimale Befriedigung der Anwenderbedürfnisse maximiert werden (vgl. Kambil et al. 1996, S. 13; Cleland/Bruno 1996, S. 3). Das Erkennen der Anwenderbedürfnisse und das Feststellen des Grades der tatsächlich erfolgten Bedürfnisbefriedigung stellen, neben der Senkung des Anwender-Aufwands, die Hauptziele des Redesign-Prozesses dar.

Was den Grad der Bedürfnisbefriedigung betrifft, so sind folgende Stufen zu unterscheiden (vgl. Albrecht 1993, S. 155ff):

Erwartete Bedürfnisbefriedigung: Erfolgt durch das WMIS nicht einmal die erwartete Bedürfnisbefriedigung, so läuft man Gefahr einen Großteil der Anwender zu verlieren. Es ist daher festzustellen, welche (Informations-) Bedürfnisse Anwender durch ein WWW-basiertes Masseninformationssystem befriedigt wissen wollen. So erwarten sich zum Beispiel potentielle Studierende von einem WWW-basierten Masseninformationssystem einer Universität, daß sie sich Basisinformation über die angebotenen Lehrprogramme abrufen können.

Erwünschte Bedürfnisbefriedigung: Die Befriedigung dieser Bedürfnisse ist zwar erwünscht, wird aber nicht gefordert oder erwartet.

1 Aus Gründen der besseren Lesebarkeit wurde auf die explizite Trennung von weiblicher und männlicher Form verzichtet. 
Unerwartete Bedürfnisbefriedigung: Dabei handelt es sich um die Befriedigung von Bedürfnissen, die dem Anwender entweder selbst nicht bewußt sind bzw. mit deren Befriedigung sie nicht gerechnet haben.

Der Grad der Bedürfnisbefriedigung ist vom WWW-Angebot der Konkurrenz sowie anderen Umweltbedingungen abhängig. Aus einer unerwarteten Bedürfnisbefriedung kann schnell eine erwartete Bedürfnisbefriedigung werden. Daher gilt es beim Redesign eines WWW-basierten Masseninformationssystems die Umwelt des WMIS genau zu analysieren. Als signifikante, umweltbedingte Einflußfaktoren von Masseninformationssystemen bezeichnet Hansen die Unternehmensbranche, die aktuelle Telekommunikationsinfrastruktur und die eigene Unternehmensstruktur (Hansen 1995, S. 129f.).

Unter Anwender-Aufwand versteht man wiederum jene Kosten, Zeit und Anstrengungen, die bei der Bedürfnisbefriedigung entstehen. Bei einem WMIS sind dies in erster Linie die Suchkosten, die durch den Suchprozeß verursacht werden. Ein Suchprozeß kann in folgende Phasen gegliedert werden: Suche nach dem WMIS selbst, Suche innerhalb des WMIS und Abrufen der Information (siehe Abb. 2).

Der Suchaufwand nach einem bestimmten WWW-basierten Masseninformationssystem innerhalb des World Wide Web kann durch die Bewerbung der Web-Site minimiert werden. Daraus läßt sich ableiten, daß die Bewerbung von WWW-basierten Masseninformationssystemen den Anwender-Nettonutzen eines Informationssystems erhöht. Auf das Thema "Bewerbung von Web-Sites" soll aber an dieser Stelle nicht näher eingegangen werden (siehe u.a. Helmstetter 1997).

Die Minimierung des Suchaufwands innerhalb des Informationssystems kann durch eine möglichst effiziente Organisation der angebotenen Information erzielt werden. Schon bei der Entwicklung des WMIS sollte die Strukturierung des WWW-Angebots auf die Anwenderbedürfnisse abgestimmt werden. In dieser Phase kann zum Beispiel anhand der Methode von Fuccella/Pizzolato vorgegangen werden (Fuccella/Pizzolato 1998) ${ }^{2}$. Beim Redesign muß dann überprüft werden, ob die aktuelle Organisation der Web-Site den Anforderungen der Anwender gerecht wird. Genauso sind beim Redesign auch irreführende Bezeichnungen von Hyperlinks ausfindig zu machen und zu eliminieren, da diese den Anwender-Aufwand genauso steigern wie eine schlechte Strukturierung der Information.

2 Dabei wird an potentielle WMIS-Anwender je ein Stapel Karten ausgeteilt. Jede Karte bezeichnet ein Informationsobjekt, das in der vorangegangenen Analysephase identifiziert wurde. Danach geht es daran, die einzelnen Karten in Gruppen zu sortieren und diese Gruppen zu bezeichnen. Aus der Organisation der Informationsobjekte können Organisationsobjekte abgeleitet und implementiert werden. 
SUCHPHASEN EINFLUSSFAKTOREN

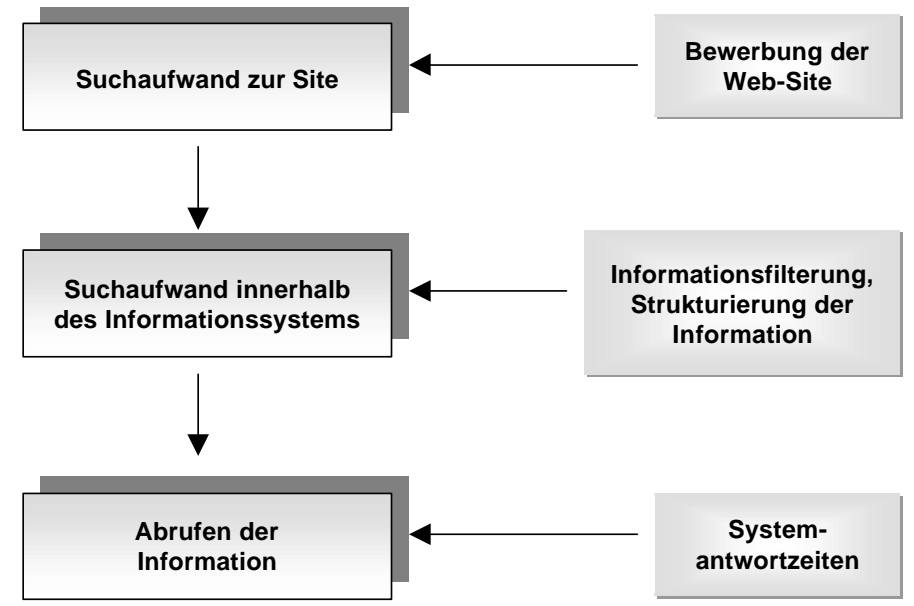

Abbildung 2: Teilphasen des Suchprozesses und Einflußgrößen auf den Anwender-Aufwand

Auf der anderen Seite kann der Suchaufwand durch vorgeschaltete, automatisierte Informationsfilter minimiert werden. Adaptive Techniken ermöglichen individualisierte, dynamisch erstellte Navigationshilfen und erhöhen dabei den Komfort bei der Benutzung des Systems. Aber auch ohne solch anspruchsvolle Applikationen kann ein WWW-basiertes Masseninformationssystem schon alleine durch die Bereitstellung von anwendergruppenspezifischen Menüs den Suchaufwand minimieren. Wird den Anwendern auch die Möglichkeit geboten, die Navigationshilfen eigenständig zu konfigurieren, so kann der Suchaufwand noch weiter reduziert werden.

Im letzen Schritt, beim Abrufen der Information aus dem WMIS, ist es vor allem die Systemantwortzeit, die sich auf den Anwender-Aufwand auswirkt. Als Einflußfaktoren auf die Systemantwortzeit können genannt werden: das Design (im engeren Sinne), WWW-Anwendungen und die Systemressourcen des Informationssystems. Andere Einflußfaktoren, wie etwa die Datenübertragungsrate zwischen Anwender und Serviceprovider, liegen oft nicht mehr im Einflußbereich des Betreibers des Informationssystems.

An dieser Stelle sei erwähnt, daß das Minimieren der Suchkosten nicht bei jeder Web-Site oberstes Ziel des Redesigns sein muß. Ähnlich wie der Konsument den Schaufensterbummel genießt, kann der Surfer das (vielleicht langwierige) Durchsuchen von Web-Sites genießen (vgl. Whinston et al. 1997, S. 268f.). Es muß daher die Entscheidung getroffen werden, ob man eine Web-Site für Surfer oder für Anwender entwickelt (vgl. Spool et al. 1997, S. 11). 
Das im folgenden präsentierte Vorgehensmodell orientiert sich an Benutzergruppen, die Information nachfragen, die sie zur Lösung eines offenen Problems benötigen. Das heißt die Zielgruppe der Surfer wird hier vernachlässigt. Das Modell dient zur Aufdeckung nutzenstiftender Information sowie der Reduzierung des Anwender-Aufwands beim Einsatz des Informationssystems (Suchphase 2 in Abb. 2). Es soll einen Leitfaden für die Durchführung eines effektiven Redesigns liefern, das auf einer gezielten Konkurrenzanalyse sowie auf der systematischen Beobachtung des Verhaltens der Besucher eines WMIS aufbaut.

\section{Das Vorgehensmodell zum Redesign von WMIS}

Um herauszufinden, ob ein Redesign nötig ist, müssen zunächst Kriterien für die Erfolgsmessung definiert werden. Dazu können folgende Daten des WMIS verwendet werden: Zugriffshäufigkeit, Anzahl der Anwender, Verweildauer pro Besucher und Anzahl der abgerufenen Dokumente pro Anwender. Die Zielvorgaben können auch von den Soll-Werten der Geschäftsprozesse abgeleitet werden. So kann zum Beispiel vom Geschäftsprozeß "Die Studierenden über JobAngebote on-line informieren" ein Erfolgskriterium für das WMIS abgeleitet werden. Aus den vom WMIS unterstützten Geschäftsprozessen können aggregierte Zielvorgaben für das gesamte WMIS ermittelt werden. Die Erfüllung der aggregierten Zielvorgaben müssen regelmäßig und über einen längeren Zeitraum hinweg überprüft werden. Ergibt die Untersuchung ein signifikant rückläufiges Ergebnis, so wird ein Redesign eingeleitet.

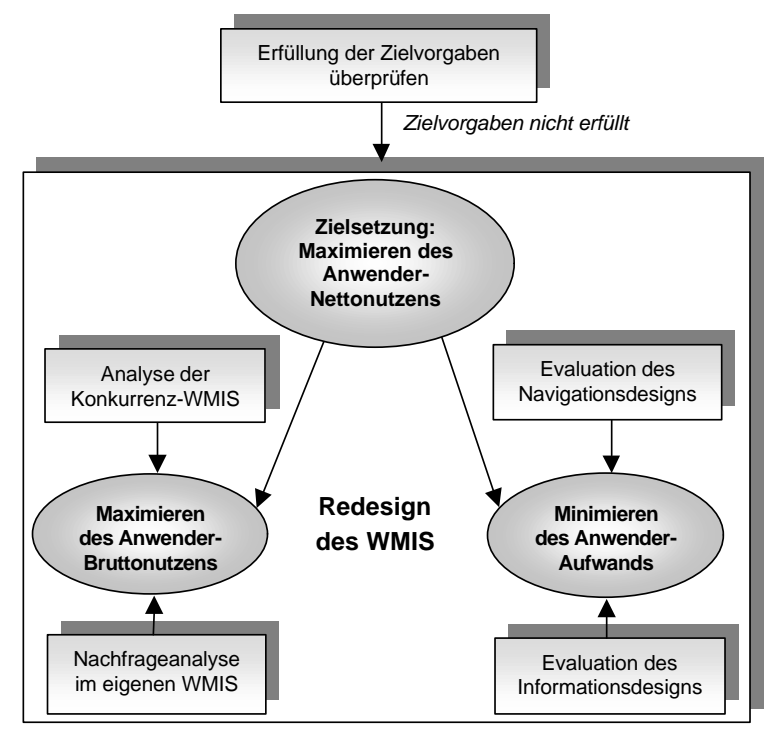


Abbildung 3: Das Vorgehensmodell zum Redesign von WMIS

Das Vorgehen beim Redesign besteht aus zwei Teilen. Um ein optimales WMIS im Sinne eines maximierten Anwender-Nettonutzens zu erhalten, muß einerseits der Anwender-Bruttonutzen maximiert und andererseits der Anwender-Aufwand minimiert werden. Das Informationsangebot des eigenen WMIS sowie das der Konkurrenz wird dabei einer genauen Prüfung unterzogen, um den AnwenderBruttonutzen steigern zu können. Dazu werden Methoden wie die Konkurrenzanalyse, das Benchmarking und die Log-File-Analyse eingesetzt. Um den Anwender-Aufwand reduzieren zu können, wird die Benutzerfreundlichkeit der Strukturierung sowie des Navigationsdesigns des WMIS evaluiert, wobei wiederum die Log-File-Analyse angewandt werden kann (siehe Abb. 3).

\subsection{Maximierung des Anwender-Bruttonutzens}

Im folgenden wird eine Möglichkeit beschrieben, wie der AnwenderBruttonutzen eines WMIS gesteigert werden kann. Zunächst wird von einer Konkurrenzanalyse auf die erforderlichen Inhalte eines WMIS geschlossen. Mit Hilfe der Auswertung von Zugriffszahlen auf einzelne Dokumente kann die Nachfragehäufigkeit der einzelnen Informationsobjekte des eigenen WMIS erhoben werden. Decken sich diese nicht mit den Zielvorgaben, so müssen die Ursachen ermittelt werden, um ein gezieltes Redesign durchführen zu können. Unter Informationsobjekten verstehen die Autoren, in Hinblick auf die einfache Auswertung mit Hilfe der Log-File-Analyse, die einzelnen am Web-Server gespeicherten Web-Dokumente.

\subsubsection{Analyse der Konkurrenz-WMIS}

Aus der Analyse der WMIS der Konkurrenz kann auf fehlende Information im eigenen WMIS geschlossen und Verbesserungspotential erkannt werden. Bei der dabei verwendeten Methode der Konkurrenzanalyse müssen die Mitbewerber identifiziert und ihre WMIS analysiert werden. Einzelne, nicht im eigenen WMIS enthaltene Inhalte werden dahingehend untersucht, ob sie in das eigene WMIS aufgenommen werden sollten.

Auch das Benchmarking spielt beim Redesign von WMIS eine Rolle. Mit Benchmarking orientieren sich Unternehmen an den Leistungen ihrer stärksten Konkurrenten. Zunächst bestimmt das Unternehmen die kritischen Erfolgskriterien seines WMIS. Danach werden die eigenen Resultate mit Spitzenleistungen der Konkurrenz verglichen (vgl. Belz 1998, S. 63). Teilweise können auch branchenfremde Anbieter als Maßstab dienen. Diese wären hier anerkannte Erfolgsunternehmen, wie Amazon.com.

Im nächsten Schritt wird mittels kreativer Methoden versucht, zusätzliche Information zu bestimmen, die von der Konkurrenz noch nicht angeboten wird, um das eigene WMIS erfolgreich von der Konkurrenz abzuheben. 


\subsubsection{Nachfrageanalyse im eigenen WMIS}

Das Interesse der Anwender an den angebotenen Informationsobjekten eines WMIS kann durch die Log-File-Analyse erhoben werden. Dazu bildet der WMIS-Betreiber geeignete Zielvorgaben über die Nachfragehäufigkeiten und vergleicht diese mit der tatsächlich gemessenen Zugriffszahl der einzelnen Informationsobjekte. Die Erwartungen basieren entweder auf den Erfahrungen aus dem traditionellen Auftreten des WMIS-Betreibers, auf Kundenbefragungen oder werden aus den Soll-Werten der Geschäftsprozesse abgeleitet.

Die Log-File-Analyse kann aufgrund der am Markt bereits vorhandenen Werkzeuge sehr effizient durchgeführt werden ${ }^{3}$. In einer Log-Datei protokolliert der Web-Server die einzelnen Anfragen der Clients. Eine Anfrage bedeutet dabei den Abruf einer Datei im Dateisystem des Server-Rechners. Der Web-Server kann Variablen wie IP-Adresse oder Hostname des Anwenders, abgefragte Datei, vermittelte Bytes, Browser und Betriebssystem des Anwenders in der Log-Datei aufzeichnen (vgl. Eilebrecht 1997, S. 147ff.). Die Log-File-Analyse selbst ermöglicht das Auswerten dieser Datei im Sinne einer Anwenderbeobachtung.

In diesem Zusammenhang müssen zwei Schwachstellen der Log-File-Analyse hervorgehoben werden: Einerseits verursachen nicht alle abgerufenen Dateien eine Serveranfrage, da Web-Browser sowie Proxy-Server Dokumente zwischenspeichern (cachen). Andererseits scheinen in der Log-Datei auch Zugriffe auf Dateien auf, die der Anwender zwar aufgerufen, aber nicht gelesen hat. Diese Schwachstelle kann durch die Definition eines Kriteriums umgangen werden, womit das Interesse des Anwenders an dem entsprechenden Objekt nachvollzogen werden kann. Dies kann zum Beispiel die Metainformation "Auffassungszeit" sein, die für jedes Informationsobjekt angibt, wie lange ein Anwender benötigt, um die Kerninformation des Objektes zu erfassen. Diese Metainformation kann mit der aus der Log-Datei extrahierten Aufenthaltsdauer verglichen werden, um gezielt abgerufene Informationsobjekte identifizieren zu können.

Entsprechen die Ergebnisse nicht den Zielvorgaben, muß in einem nächsten Schritt herausgefunden werden, ob diese überhöht waren. Dies kann zum Beispiel durch die Ausrichtung an einer falsche Zielgruppe eintreten. Die Ursache kann aber auch auf einen zu hohen Anwender-Aufwand bei der Benutzung des WMIS zurückgeführt werden.

\subsection{Minimierung des Anwender-Aufwands}

Der Anwender-Aufwand entsteht beim Versuch der Anwender, mit Hilfe des WMIS ihre offenen Informationsbedürfnisse zu decken bzw. ein bestimmtes

3 http://www.webtrends.com/ http://www.nettracker.com/products/NetTracker/ http://www.marketwave.com/products.htm 
Problem zu lösen. Dieser hängt dabei hauptsächlich von der anwenderfreundlichen Gestaltung der Benutzerschnittstelle des Systems ab.

Eine optimale Benutzerschnittstelle ist dann gegeben, wenn sie "benutzbar" ist und den Anwender bei der Lösung seiner Probleme mit Hilfe des Systems optimal unterstützt. Dafür hat sich der Begriff "Usability" eingebürgert. Nach Shackel hat Usability vier Hauptaspekte: Effektivität, Lernbarkeit, Flexibilität und Einstellung der Anwender (Shackel zitiert in Redmond-Pyle et al. 1995, S. 3). Die Autoren fokussieren ihre Analyse auf die Effektivität der Benutzerschnittstelle. Effektivität bedeutet hier die Möglichkeit des Systems, den Anwender dabei zu unterstützen, seine Aufgabe produktiv zu erfüllen und bezieht sich auf die Schnelligkeit, mit der die Anwender ihre Aufgaben erfüllen können (Redmond-Pyle et al. 1995, S. 3). Dieses Kriterium läßt sich einfach quantifizieren und messen. Zwei allgemeine Ansätze, Usability zu messen, sind (Redmond-Pyle et al. 1995, S. 4):

a) Leistungstests, bei denen die Anwender das System einsetzen, um bestimmte Aufgaben zu erfüllen und dabei die Effektivität in Form von Zeit- und Fehlermessungen erhoben wird und

b) Akzeptanzstudien, bei denen die Anwenderzufriedenheit und Anwenderwahrnehmung mit Hilfe von Fragebögen und Interviews erhoben wird.

Die Autoren beschränken ihre Untersuchung auf die Log-File-Analyse, die dazu eingesetzt wird, die Effektivität der Benutzerschnittstelle eines WMIS zu messen. Neben der Log-File-Analyse können auch andere Methoden herangezogen werden, wie zum Beispiel die Thinking-Aloud-Methode (Redmond-Pyle et al. 1995, S. 257).

Um den Anwender-Aufwand eines WMIS zu senken, wird die Effektivität der Benutzerschnittstelle des WMIS untersucht. Um eine Evaluation nach RedmondPyle (Redmond-Pyle et al. 1995) durchführen zu können, müssen zunächst Usability-Requirements definiert werden. Mit deren Hilfe können Meßinstrumente und Meßwerte eingesetzt werden, die die Quantifizierung und Nachmeßbarkeit der Effektivität ermöglichen. Dabei werden für jedes Meßkriterium Bandbreiten der geplanten, bestmöglichen und mindestakzeptablen Ergebnisse eingesetzt. Die Resultate der Evaluierung decken schließlich die Schwachstellen des Systems auf. So kann das Usability-Requirement "Effektive Strukturierung und Linkbenennung" wie folgt evaluiert werden:

a) Meßinstrument: Erreichbarkeit der am häufigsten nachgefragten Information.

Meßwert: Überprüfung des eingeschlagenen Weges zur Information.

Auswertung: Vergleich mit dem kürzest möglichen Weg zur Information.

b) Meßinstrument: "Verlorengehen" innerhalb der Site.

Meßwert 1: Auffinden von Objekten, an denen das WMIS überdurchschnittlich oft verlassen wurde (Exit-Punkte der Site). 
Meßwert 2: Aufdecken von Objekten, von denen immer wieder zu einem Hauptorientierungspunkt zurückgekehrt wird.

Auswertung: Die Ausstiegshäufigkeit übersteigt einen kritischen Wert.

c) Meßinstrument: Unerwartete Zusammenhänge zwischen Informationsobjekten identifizieren.

Meßwert: Auffinden von Informationsobjekten, die von überdurchschnittlich vielen Anwendern während einer Sitzung aufgerufen werden und in verschiedenen Kategorien angeordnet sind.

Auswertung: Die Anzahl der Informationsobjekte, die während einer Sitzung in einer nicht vom WMIS unterstützten Reihenfolge aufgerufen wurden, übersteigt einen kritischen Wert.

Im Rahmen der Aufwandsmessung von Benutzerschnittstellen WWW-basierter Masseninformationssysteme wird zwischen dem Informationsdesign und dem Navigationsdesign unterschieden. Powell identifiziert noch eine dritte Kategorie, nämlich das Applikationsdesign, das wir hier jedoch vernachlässigen wollen (Powell 1998, S. 151ff.). Informationsdesign bezieht sich auf die Struktur des WMIS und beschreibt die Organisation der Informationsobjekte. Das Navigationsdesign bezieht sich im text-basierten Kontext auf die Eindeutigkeit der Bezeichnung von Hyperlinks. Die Struktur des WMIS und die Bezeichnung der Hyperlinks können nicht einfach getrennt voneinander evaluiert werden. Die Ursache von Bedienungsfehlern seitens der Anwender kann meist nicht eindeutig einer der beiden Kategorien zugeordnet werden. Vor allem bei der Log-FileAnalyse ist eine solche Differenzierung nicht möglich.

Im folgenden werden Informations- und Navigationsdesign getrennt analysiert.

\subsubsection{Evaluation des Informationsdesigns (Strukturierung des WMIS)}

Die Strukturierung eines WMIS dient dazu, Klarheit, Bedeutung und Kontext der Information zu bieten.

Um ein WMIS effektiv zu strukturieren, ist es wichtig, die Hypertexttheorie und verschiedene Hypertextmodelle zu verstehen. Shneiderman definiert drei Grundregeln für die Gestaltung von Hypertext-Systemen (Shneiderman 1992, S. $410 \mathrm{f}$.):

a) Ein großer Informationskörper wird in viele verschiedene Segmente gegliedert, die im Fall eines WMIS einzelne Informationsobjekte sind.

b) Die Seiten beziehen sich aufeinander.

c) Der Anwender benötigt nur einen kleinen Teil der Information zu einem bestimmten Zeitpunkt.

Die "Mixed-Hierarchy"-Strukturierung (Powell et al. 1998, S. 146) ist die üblichste Form der Hypertextstrukturierung. Sie verzweigt als "hierarchische Strukturierung" von einer allgemeinen Übersicht auf der obersten Ebene (Homepage) bis $\mathrm{zu}$ vielen spezifischen Dokumenten in den unteren Hierarchieebenen. Zusätzlich bietet diese Strukturierungsmethode die 
Möglichkeit, Hierarchiestufen auszulassen und direkt auf untere Ebenen zu springen (siehe Abb. 4).

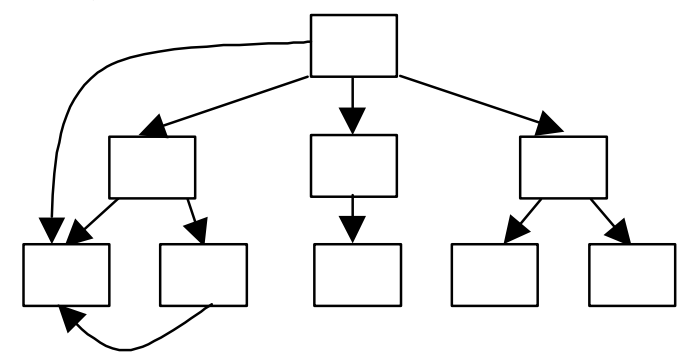

Abbildung 4: Mixed-Hierarchy-Strukturierung

Häufig werden für die Strukturierung falsche Ansätze gewählt, wie die Strukturierung nach der Aufbauorganisation des Web-Site-Betreibers (vgl. u.a. Nielsen 1998, n.p.). Wenn die Struktur eines WMIS unklar ist, wird es für den Anwender schwierig, sich zu orientieren, was zu einer homepage-basierenden Navigation führt (Powell et al. 1998, S. 147). Dabei wurde beobachtet, daß Anwender, die verloren gehen, zu einem ihnen bekannten Punkt zurückkehren wollen, der meistens die Homepage des WMIS ist.

Probleme bei der Navigation treten vor allem dann auf, wenn die Struktur des WMIS Bereichskenntnisse voraussetzt oder nicht den Erwartungen des Anwenders entspricht. Es muß daher hinterfragt werden, ob bei der Navigation im WMIS nicht eventuell spezielle Kenntnisse vom Anwender erwartet werden, die dieser jedoch nicht besitzen kann (Spool et al. 1997, S. 13). Anwender wollen nicht lange klicken, bis sie zur relevanten Information gelangen (Shneiderman 1992, S. 411). Wird bestimmten Informationsobjekten ein hoher Nutzen zugeordnet, dann sollte diese Information leicht erreichbar angeordnet sein (vgl. Buchanan et al. 1997, S. 107).

Die Kerninformation eines WMIS wird im präsentierten Modell anhand der Zugriffshäufigkeit erhoben. Das Abrufen der Kerninformation durch den Anwender sollte immer möglichst wenig Anwender-Aufwand verursachen. Um dies zu erzielen, sollte diese Information in einem Mixed-Hierarchy-Design von hohen Hierarchieebenen aus schnell erreichbar sein. Da sich die Anwenderbedürfnisse und damit das Nachfrageverhalten laufend ändern, bringt ein flexibles System große Vorteile. Dabei kann die Anordnung der Hyperlinks automatisch abhängig von der Zugriffshäufigkeit erfolgen.

\subsubsection{Evaluation des Navigationsdesigns}

Das Navigationsdesign dient zur Unterstützung der Interaktion des Anwenders mit dem System. Powell unterscheidet drei Möglichkeiten der Navigationsunterstützung: text-, grafik- und methaphern-basiert (Powell 1998, S. 162). Da wir in unserem Beitrag grafische Kriterien nicht behandeln, 
konzentrieren wir uns auf die text-basierte Navigationsunterstützung. LayoutKriterien, wie zum Beispiel Farb- und Leerraumeinsatz (siehe u.a. Mullet et al. 1995), die ebenfalls zur Aufwandsreduktion der Anwender beim Auffinden von Information dienen, werden außer acht gelassen. Ein text-basiertes Navigationsdesign bietet zur Ermöglichung der Navigation nur Hyperlinks, die durch Text dargestellt werden. Wichtig ist in diesem Zusammenhang vor allem die Benennung der Hyperlinks.

Der Erfolg der Bezeichnung der Hyperlinks hängt davon ab (Spool et al. 1997, S. 31),

a) wie gut er dem Anwender erlaubt, vorherzusagen, wohin der Link führt und

b) wie gut er von anderen, nahegelegenen Links unterschieden werden kann.

Es können auch zusätzliche Hinweise zu Hyperlinks angebracht werden, wie die spezielle Kennzeichnung von neu verfügbaren Informationsobjekten. Je besser die Anwender vorhersehen können, wohin ein Link führt, um so erfolgreicher werden sie beim Auffinden von Information unterstützt und um so benutzerfreundlicher wird das System wahrgenommen.

Da die verschiedenen Navigationswege durch ein Informationssystem nicht vorhergesehen werden können, ist es vorteilhaft, sogenannte "Supplemental Navigation Systems" vorzusehen, wie zum Beispiel Suchmaschinen, Site-Maps oder Inhaltsverzeichnisse ${ }^{4}$. Auch diese können anhand einer Log-File-Analyse auf ihre Benutzerfreundlichkeit untersucht werden.

\subsection{Redesign}

Das Ergebnis des gesamten Prozesses ist das eigentliche Redesign, das die Umsetzung folgender Änderungen umfassen kann:

a) Das Integrieren neuer Informationsobjekte in das WMIS: Dies ergibt sich aus der Konkurrenzanalyse oder aus dem genaueren Verstehen der Informationsbedürfnisse der Anwender, das im Rahmen der Anwenderbeobachtung erreicht werden konnte.

b) Das Umbenennen der Hyperlinks: Dies kann das Ergebnis der Evaluation der Benutzerschnittstelle sein.

c) Das Umstrukturieren der Site: Dies kann ebenfalls das Ergebnis der Evaluation der Benutzerschnittstelle sein. Dabei werden aufgedeckte Schwachstellen der Strukturierung beseitigt, indem

- Informationsobjekte anderen, bereits vorhanden Kategorien zugeordnet werden,

- zusätzliche Links aus weiteren Kategorien eingefügt werden, da die Anwender zum Beispiel auf dem Weg nach Informationsobjekt $\mathrm{X}$ auffallend oft die Kategorie Y aufgerufen haben, oder

- neue Kategorien gebildet werden.

4 Siehe u.a. http://webreview.com/97/10/03/arch/ 
Der Erfolg der realisierten Maßnahmen muß wiederum anhand der festgelegten Zielvorgaben überprüft werden (siehe Abb. 3). 


\section{Anhang A: Gegenüberstellung von Customer Value- Definitionen}

\begin{tabular}{|l|l|l|}
\hline Autor & Bezeichnung & Definition \\
\hline $\begin{array}{l}\text { Bradley 1994, } \\
\text { S. XIV }\end{array}$ & Customer Value & $\begin{array}{l}\text { Customer value = } \\
\text { Market-perceived quality / Price }\end{array}$ \\
\hline Cleland/Bruno 1996, S. 3 & Customer Value & $\begin{array}{l}\text { Customer value = } \\
\text { Quality (product + non-product quality) / Price }\end{array}$ \\
\hline $\begin{array}{l}\text { Kambil 1996, } \\
\text { S. 13 }\end{array}$ & $\begin{array}{l}\text { Customer Value } \\
\text { (Value Proposition) }\end{array}$ & $\begin{array}{l}\text { Customer value } \\
\text { (across multiple customer roles) }= \\
\text { Performance } \\
\text { (product attributes matching needs) - } \\
\text { Costs }\end{array}$ \\
\hline $\begin{array}{l}\text { Kotler 1994, } \\
\text { S. 552 }\end{array}$ & $\begin{array}{l}\text { Customer Delivered } \\
\text { Value }\end{array}$ & $\begin{array}{l}\text { Customer delivered value = } \\
\text { Total customer value - }\end{array}$ \\
\hline $\begin{array}{l}\text { Naumann 1995, customer costs } \\
\text { S. 16 ff }\end{array}$ & $\begin{array}{l}\text { Customer Value } \\
\text { Kaukal/Simon }\end{array}$ & $\begin{array}{l}\text { Value-based prices - Product Quality - } \\
\text { Service Quality }\end{array}$ \\
\hline & Anwender- & $\begin{array}{l}\text { Anwender-Nettonutzen = } \\
\text { Anwender-Bruttonutzen (Bedürfnisbefriedigung } \\
\text { durch das Informationsangebot des WMIS) - } \\
\text { Anwender-Aufwand }\end{array}$ \\
\hline
\end{tabular}

\section{Literaturverzeichnis}

Albrecht, K. (1993): Total Quality Service - Das einzige, was zählt. Düsseldorf, et al. 1993.

Belz C. (1998): Akzente im innovativen Marketing. St. Gallen 1998.

Bichler, M. (1997): Aufbau unternehmensweiter WWW-Informationssysteme. Braunschweig/Wiesbaden 1997.

Bradley, G. T. (1994): Managing customer value : creating quality and service that customers can see. New York City 1994.

Buchanan R. W./Lukaszewski C. (1997): Measuring the impact of your Web site. New York et al. 1997.

Cleland, A. S./Bruno, A. V. (1996): The market value process : bridging customer and shareholder value. San Francisco 1996.

Eilebrecht L. (1997): Apache Web-Server. Bonn 1997.

Fuccella, J./Pizzolato J. (1998): Creating Web site designs based on user expectations and feedback. ITG Newsletter (1998)6. 
Hansen, H. R. (1995): Conceptual framework and guidelines for the implementation of mass information systems. In: Information \& Management 28(1995), S. 125-142.

Hansen, H. R. (1996): Wirtschaftsinformatik I. 7. Aufl., Stuttgart 1997.

Helmstetter, G. (1997): Increasing hits and selling more on your Web-Site. New York, et al. 1997.

Kambil, A./Ginsberg, A./Bloch, M. (1996): Re-inventing Value Propositions. Working Paper Series (Stern \#IS-96-21). New York City 1996: New York University - Stern School of Business.

Kotler, P./Armstrong, G. (1994): Principles of Marketing. 6. Aufl. London, et al. 1994.

Mullet, K./Sano, D. (1995): Designing visual interfaces. Englewood Cliffs 1995.

Naumann, E. (1995): Creating customer value: the path to sustainable competitive advantage. Cincinnati 1995.

Nielsen, J. (1998): The alertbox: current issues in web usability http://www.useit.com/alertbox/.

Österle, H. (1995): Business Engineering - Prozeß- und Systementwicklung. Berlin, et al. 1995.

Powell, T. A./Jones, D. L./Cutts D. C. (1998): Web-Site Engineering - Beyond Web Page Design. Upper Saddly River 1998.

Redmond-Pyle D./Moore A. (1995): Graphical user interface design and evaluation. London et al. 1995.

Shneiderman B. (1992): Designing the user interface - Strategies for effective human-computer interaction. $2^{\text {nd }}$ ed., Reading et al. 1992.

Spool, J. M. et al. (1997): Web site usability: a designer's guide. North Andover 1997.

Whinston, A. B./Stahl, D. O./Soon-Yong, C. (1997): The economics of electronic commerce. Indianapolis 1997. 